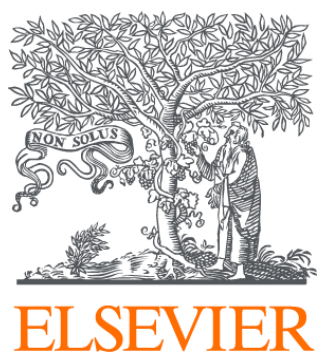

Since January 2020 Elsevier has created a COVID-19 resource centre with free information in English and Mandarin on the novel coronavirus COVID-

19. The COVID-19 resource centre is hosted on Elsevier Connect, the company's public news and information website.

Elsevier hereby grants permission to make all its COVID-19-related research that is available on the COVID-19 resource centre - including this research content - immediately available in PubMed Central and other publicly funded repositories, such as the WHO COVID database with rights for unrestricted research re-use and analyses in any form or by any means with acknowledgement of the original source. These permissions are granted for free by Elsevier for as long as the COVID-19 resource centre remains active. 


\title{
Examining SARS-CoV-2 Interventions in Residential Colleges Using an Empirical Network
}

\author{
Hali L. Hambridge ${ }^{a, *}$, Rebecca Kahn ${ }^{b}$, Jukka-Pekka Onnela ${ }^{a}$ \\ a Department of Biostatistics, Harvard T.H. Chan School of Public Health, Boston, MA, United States \\ ${ }^{\mathrm{b}}$ Center for Communicable Disease Dynamics, Harvard T.H. Chan School of Public Health, Boston, MA, United States
}

\section{A R T I C L E I N F O}

\section{Article history:}

Received 3 August 2021

Revised 17 September 2021

Accepted 2 October 2021

\section{Keywords:}

SARS-CoV-2

COVID-19

Repeat testing

Copenhagen Network Study

Proximity network

Bluetooth

\begin{abstract}
A B S T R A C T
Objectives Universities have turned to SARS-CoV-2 models to examine campus reopening strategies. While these studies have explored a variety of modeling techniques, none have used empirical data.

Methods In this study, we use an empirical proximity network of college freshmen obtained using smartphone Bluetooth to simulate the spread of the virus. We investigate the role of immunization, testing, isolation, mask wearing, and social distancing in the presence of implementation challenges and imperfect compliance.
\end{abstract}

Results We show that frequent testing could drastically reduce the spread of the virus if levels of immunity are low, but its effects are limited if immunity is more ubiquitous. Furthermore, moderate levels of mask wearing and social distancing could lead to additional reductions in cumulative incidence, but their benefit decreases rapidly as immunity and testing frequency increase. However, if immunity from vaccination is imperfect or declines over time, scenarios not studied here, frequent testing and other interventions may play more central roles.

Conclusions Our findings suggest that although regular testing and isolation are powerful tools, they have limited benefit if immunity is high or other interventions are widely adopted. If universities can attain even moderate levels of vaccination, masking, and social distancing, they may be able to relax the frequency of testing to once every four weeks.

Published by Elsevier Ltd on behalf of International Society for Infectious Diseases. This is an open access article under the CC BY license (http://creativecommons.org/licenses/by/4.0/)

\section{Introduction}

When SARS-CoV-2 escalated to a pandemic in early 2020, universities and colleges around the world were forced to rapidly pivot to virtual instruction. Schools struggled to adapt to a new normal, sending students home as residential campuses, and even entire cities, were locked down to stop the spread of SARS-CoV-2. Studies have shown that the rapid shift to virtual instruction exacerbated pre-existing educational and social inequities, leading to learning losses and leaving students feeling unmotivated (Shin and Hickey, 2021). As the pandemic continued into the summer, universities were faced with a difficult choice: reopen campuses with some return to traditional in-person instruction in order to provide a rich educational experience or continue teaching entirely online to protect the health of students.

\footnotetext{
* Corresponding author.

E-mail addresses: hhambridge@g.harvard.edu (H.L. Hambridge), rek160@mail.harvard.edu (R. Kahn), onnela@hsph.harvard.edu (J.-P. Onnela).
}

In the autumn of 2020 , college administrators around the world employed simulations to understand how enhanced public health protocols could mitigate the spread of SARS-CoV-2 on their campuses. These studies examined various preventive techniques and made different assumptions about compliance with their proposed policies (Brook et al., 2020; Chang et al., 2020; Christensen et al., 2020; Elbanna et al., 2020; Ghaffarzadegan et al., 2020; Goyal et al., 2021; Gressman and Peck, 2020; Lopman et al., 2021; Paltiel et al., 2020). However, although various modeling techniques were employed, from compartmental homogeneous mixing models to contact networks to agent-based models, all of these studies only used simulated data.

In their review of COVID-19 modeling studies in a university setting, Christensen et al. advocated for more research to be done using empirical mixing data (Christensen et al., 2020). Here we take up that charge and examine how using a real-world contact network of students on a college campus, ascertained using smartphone Bluetooth data, changes our understanding of the role of various interventions in the mitigation of SARS-CoV-2. As colleges 
look to reopen in the autumn of 2021, they will need to navigate a rapidly changing environment and contend with both more transmissible variants and lower than hoped for vaccine coverage. Universities will need to identify an appropriate approach for their specific student population, one which will likely involve a complex interplay of interventions, from immunization to repeat testing and isolation to non-pharmaceutical interventions, like mask wearing and social distancing. This study seeks to equip universities with the necessary information and tools to navigate this complicated decision-making process. While we present a range of scenarios here, our study serves as a framework for universities to explore the effectiveness of different intervention strategies in their own unique setting. Our well-documented code, together with the publicly available data used for this study, allows universities to specify their own parameter values to examine scenarios that were not considered in our study. Administrators and public health officials alike can use our flexible framework to help craft evidence-based policies designed to mitigate the spread of COVID19 on college campuses.

\section{Methods}

\subsection{Proximity network}

We employ a close proximity interaction network from the Copenhagen Networks Study (CNS), which enrolled students from the Technical University of Denmark. In particular, we focus exclusively on Bluetooth proximity data acquired from 706 students, which were made publicly available in December 2019 (Sapiezynski et al., 2019). Loaner smartphones were issued to study participants who agreed to use the device as their primary phone for the duration of the study. Devices were configured to be Bluetooth discoverable at all times and to scan for nearby devices every five minutes, recording the device ID, a timestamp, and an indicator of received signal strength (RSSI), which roughly correlates with physical distance. Bluetooth proximity data are available for 28 days starting in February 2014. In order to model a typical university semester, we loop through the CNS data four times, thereby simulating a total of 16 weeks of interaction data.

\subsection{Epidemic model}

To model the spread of SARS-CoV-2, we use a discrete-time stochastic susceptible-exposed-infectious-recovered (SEIR) individual model, with separate states for symptomatic and asymptomatic cases. Each day, individuals advance to the next compartment or remain in their current one probabilistically. Each person-toperson interaction is treated as an independent event, such that the probability of being exposed increases linearly with the number of interactions with an infectious individual. We allow for presymptomatic transmission, as there is a two day period between infectiousness and symptom onset, as well as entirely asymptomatic transmission for asymptomatic cases. The parameter values are summarized in Table 1 . We investigate low, medium, and high transmission scenarios, which roughly correspond to $R_{0}$ values of $1.5,3.0$, and 4.5 , respectively. We explore a range of values as estimates of $R_{0}$ vary widely across studies and settings (Alimohamadi et al., 2020). The supplement and Figure 5 show additional details of our SARS-CoV-2 transmission model.

In addition to infection via the contact network, we allow each individual to develop infection due to outside exposure (e.g., in the broader community), regardless of their contact network interactions. We explore high and low levels of community transmission to examine the impact of community prevalence and varying levels of integration between the campus and the community. Given the potential for continued community spread in the absence of very high vaccination rates (COVID-19 Scenario Modeling Hub, 2021) and the increased transmissibility of new variants (Davies et al., 2021), we focus on a setting with higher levels of community transmission. The results obtained for the high community transmission scenario are presented in the following, and those for our low community transmission scenario are given in the supplement.

\subsection{Initial immunity}

While many countries have approved vaccines against SARSCoV-2 and are rushing to make them available to their residents, the Council on Foreign Relations estimates that it will take years for the majority of the world's population to become immunized against the virus (Felter, 2021). As such, we explore a wide range of immunization scenarios, ranging from no immunization to $80 \%$ of the student population immunized, either due to prior infection or vaccination. For simplicity, we assume perfect immunity in our model.

\subsection{Testing and isolation}

We consider symptomatic testing and scheduled testing in our simulations, and assume that both were done via polymerase chain reaction (PCR). Symptomatic testing occurs when a student seeks a test after experiencing symptoms. In order to account for individuals who may experience non-COVID related flu-like symptoms, a fraction of non-infectious individuals also present for symptomatic testing each day. Under scheduled testing, every member of the population is tested regularly in an effort to identify additional cases that would otherwise go undetected. Individuals are tested at regular intervals so the time between tests is constant for each student, although a small fraction of the population is set to be non-compliant each day. Both types of testing incorporate timedependent sensitivity; SARS-CoV-2 test sensitivity is highly correlated with viral load, which varies over the course of the infection. To account for this, we use the empirical test sensitivity derived from nasopharyngeal swab data by Wikramaratna et al. (Wikramaratna et al., 2020). Since their model only included the time after symptom onset, we impute pre-symptomatic test sensitivity, mirroring the post-symptomatic sensitivity so that the sensitivity two days prior to symptom onset is the same as that two days after symptom onset. This is consistent with literature showing that viral load peaks at symptom onset, with similar loads preand post-symptom onset (Johansson et al., 2021). Additional details are provided in the supplement and Figure 6. Upon testing positive, individuals are placed in isolation. For each set of parameter values, we examine scheduled testing frequencies of three, seven, 14 , and 28 days, as well as no scheduled testing.

\subsection{Mask wearing and social distancing}

We also consider a setting where student behavior leads to individual-specific transmission rates. We specify a proportion of the population that would wear face masks and randomly assign a subset of the population to abide by social distancing. Both interventions are implemented as reductions in the transmission rate, and these reductions are assumed to be linear. Since homophily, the tendency for people to associate with others whom they perceive to be similar to themselves, is typically present in social networks (McPherson et al., 2001), we hypothesized that friend groups might share similar views about COVID-19 and related mitigation efforts. Therefore, we consider both clustered and nonclustered assignment of mask wearing across the network, a scenario that cannot be studied with standard epidemiological models. To create clusters of mask users, we create a weighted contact network for the entire study period and select a set of initial 
Table 1

Parameters for simulation scenarios. The transmission probability, $\beta$, is per five-minute interaction with an infectious individual. All other epidemic transition parameters are per day, giving an average latent period of three days and an average infectious period of seven and 12 days for asymptomatic and symptomatic cases, respectively. Test sensitivity is time-varying. For distributions, values represent the means and standard deviations, respectively. An overview of the epidemic model is shown in Figure 5. Additional details are presented in the corresponding sections of the supplement.

\begin{tabular}{|c|c|c|}
\hline & & Epidemic Model \\
\hline$\beta$ & $0.003-0.009$ & probability of transmission per five-minute interaction \\
\hline$\pi_{e x \_h i}$ & $\operatorname{Normal}(0.002,0.0001)$ & probability of external infection per day - high scenario \\
\hline$\pi_{\text {ex_lo }}$ & $\operatorname{Normal}(0.0005,0.0001)$ & probability of external infection per day - low scenario \\
\hline$\pi_{a i}$ & 0.3 & probability of asymptomatic infection \\
\hline$\left|I_{0}\right|$ & 1 & number of initial infections \\
\hline$\sigma_{a}$ & $1 / 3$ & transition probability: exposed to asymptomatic infectious \\
\hline$\sigma_{s}$ & $1 / 3$ & transition probability: exposed to symptomatic infectious \\
\hline$\gamma_{a}$ & $1 / 7$ & transition probability: asymptomatic infectious to recovered \\
\hline \multirow[t]{2}{*}{$\gamma_{s}$} & $1 / 12$ & transition probability: symptomatic infectious to recovered \\
\hline & & Testing \\
\hline$\pi_{s e}$ & $0-0.96$ & sensitivity, time-varying \\
\hline$\pi_{s p}$ & 0.99 & specificity \\
\hline$\tau_{s y}$ & 1 day & delay between symptom onset and symptomatic testing \\
\hline$\pi_{s c}$ & $\operatorname{Normal}(0.025,0.01)$ & probability non-compliant with scheduled testing \\
\hline$\pi_{s y}$ & $\operatorname{Normal}(0.25,0.1)$ & probability non-compliant with symptomatic testing \\
\hline \multirow[t]{2}{*}{$\pi_{f s}$} & 0.005 & probability non-infectious present for symptomatic testing \\
\hline & & Isolation \\
\hline$\tau_{i d}$ & 1 day & delay between testing and entering isolation \\
\hline$\pi_{i c}$ & $\operatorname{Beta}(0.91,0.11)$ & isolation compliance \\
\hline \multirow[t]{2}{*}{$\tau_{i p}$} & 10 days & isolation period \\
\hline & & Transmission Mitigation \\
\hline$\eta_{f m}$ & $\operatorname{Normal}(0.15,0.0684)$ & reduction in transmission probability for mask wearing \\
\hline$\eta_{s d}$ & $\operatorname{Normal}(0.18,0.0734)$ & reduction in transmission probability for social distancing \\
\hline$\pi_{f m}$ & $0-1$ & proportion of the population wearing face masks \\
\hline$\pi_{s d}$ & $0-1$ & proportion of the population social distancing \\
\hline$\pi_{i m}$ & $0-0.8$ & proportion of the population immune \\
\hline
\end{tabular}

mask wearers uniformly at random. We then "spread" mask wearing to their contacts, where neighbors with more interactions have a higher probability of adopting a face covering. This process is iterated until the desired proportion of mask wearers is reached. More details are provided in the supplementary information.

\section{Results}

After removing non-participating devices and empty scans from the Bluetooth data, 2,426,279 Bluetooth pings (44.3\% of all pings) and 692 users (98.0\% of all users) remained, indicating that 14 users had no proximity events involving other study participants. The proximity networks for each of the 28 days considered in this study are shown in Figure 3 in the supplement. The networks have a large connected component on weekdays when students were likely active on campus and attending classes. On the weekends, the networks were more loosely connected, with fewer users interacting with fellow study participants, as shown in the first and last columns in Figure 3.

The incidence rates for our moderate $\left(R_{0} \approx 3.0\right)$ transmission scenario with various levels of the population immune from the outset are shown in Figure 1. Regardless of immunization level, increasing the testing frequency reduced the number of infections observed over the course of the semester, although the effect was relatively small if $60-80 \%$ of the population was immune. Under no immunization, testing every three days resulted in $42.7 \%$ of the students being infected by the end of the semester on average, whereas testing every seven, 14 , and 28 days led to $47.5 \%, 56.2 \%$, and $61.6 \%$ of students infected, respectively (no scheduled testing: $66.1 \%$ ). However, with $80 \%$ immunized, testing every three days resulted in $12.3 \%$ of students infected by the end of the semester on average, with no scheduled testing giving rise to $15.8 \%$ of stu- dents infected on average. Thus, while increased testing and subsequent isolation impacted the number of infections for a given immunization level, ultimately increasing the population immunity led to commensurate or greater reductions in incidence. Incidence for our low $\left(R_{0} \approx 1.5\right)$ and high $\left(R_{0} \approx 4.5\right)$ transmission scenarios are shown in the supplement (see Figures 9 and 10).

Under no immunization, the average time required to infect $10 \%$ of all students was 24 days without any testing or isolation, whereas testing just once every four weeks increased this time to 25 days, and testing twice each week resulted in $10 \%$ of students being infected on day 30 on average (Figure 1). However, when $20 \%$ of the population was immune, testing every three days could extend this period by 11 days, and with $40 \%$ of the population immune, frequent testing could spread infections out even further, thereby giving universities an additional two weeks. These findings demonstrate that when combined with regular testing, even modest levels of immunity can help universities flatten the curve, ultimately saving resources and reducing the cost of mitigation efforts.

The number of positive tests and number of people isolated are shown in Figures 7 and 8, respectively. These metrics allow universities to estimate the resources required to mitigate the spread of COVID-19 among their student population in terms of resources spent on interventions and lost opportunity costs. For instance, the number of students isolated over the course of the semester also corresponds to the number of missed school days, allowing universities to quantify both the resources needed to quarantine students and the lost educational opportunities associated with quarantine. Further details are provided in the supplement.

The results obtained for our mask wearing and social distancing scenarios under moderate transmission $\left(R_{0} \approx 3.0\right)$ are presented in Figure 2, which shows the mean percentage of the student pop- 


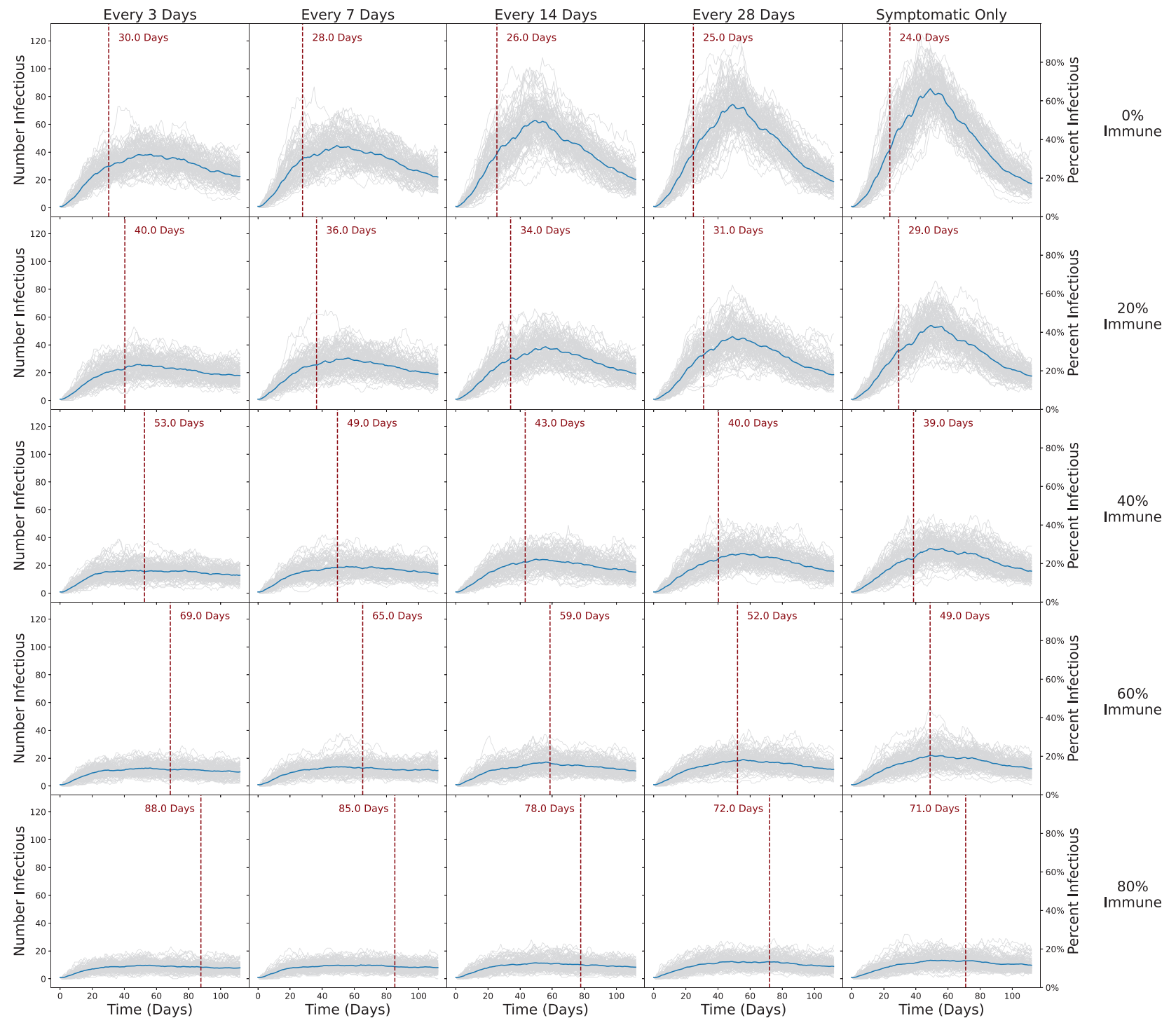

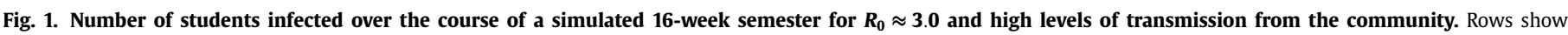

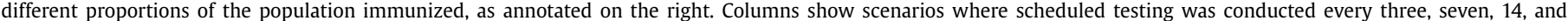

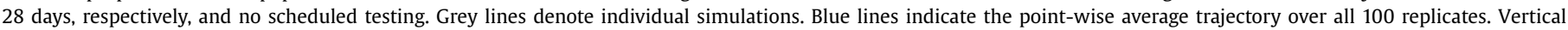

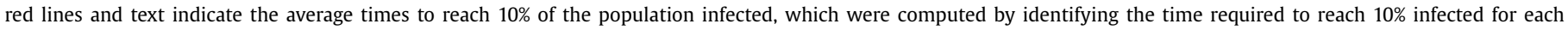
realization and averaging those times.

ulation infected at the end of the semester. Testing was most effective at reducing cumulative incidence under low levels of immunity and low to moderate levels of mask wearing and social distancing. When mask wearing and social distancing were ubiquitous, more frequent testing offered only small decreases in the cumulative incidence as the virus was already well controlled. Similarly, initial immunity in even a small fraction of the population reduced the impact of regular testing. Under no immunity, testing every three days resulted in sizeable benefits when mask wearing and social distancing were below $60 \%$. However, with $20 \%$ and $40 \%$ immune, testing every three days only provided sizeable benefits when mask wearing and social distancing were below $40 \%$ and $30 \%$, respectively. Somewhat surprisingly, little to no decrease in efficacy occurred when mask wearing was clustered on the contact network, perhaps due to the highly connected nature of the network (see Figure 11). If universities can achieve even moderate levels of immunity, mask wearing, and/or social distancing, our simulations demonstrate that they may be able to test less frequently with little or no change in the cumulative incidence.

To compare the effectiveness of testing and isolation, mask wearing, social distancing, and immunity, we conducted a regres- sion analysis based on the cumulative incidence over all of our moderate transmission simulations $\left(R_{0} \approx 3.0\right)$. The cumulative incidence decreased by 3.95 or 0.57 per 100 students for every one week increase in the testing frequency. Every $10 \%$ increase in the proportion of the population conforming to social distancing reduced the cumulative incidence by 4.47 or 0.65 per 100 students. Likewise, each $10 \%$ increase in the proportion wearing masks reduced the cumulative incidence by 5.76 or 0.83 per 100 students. Finally, each $10 \%$ increase in the proportion with immunity decreased the cumulative incidence by 17.08 or 2.47 per 100 students. Thus, while testing might be straightforward for colleges to enforce, it provides much smaller reductions in cases than mask wearing and social distancing. As expected, increasing population immunity resulted in the greatest reduction in cumulative incidence.

\section{Discussion}

While the past year has given us the promise of effective vaccines and greater insight into the dynamics of SARS-CoV-2, schools 


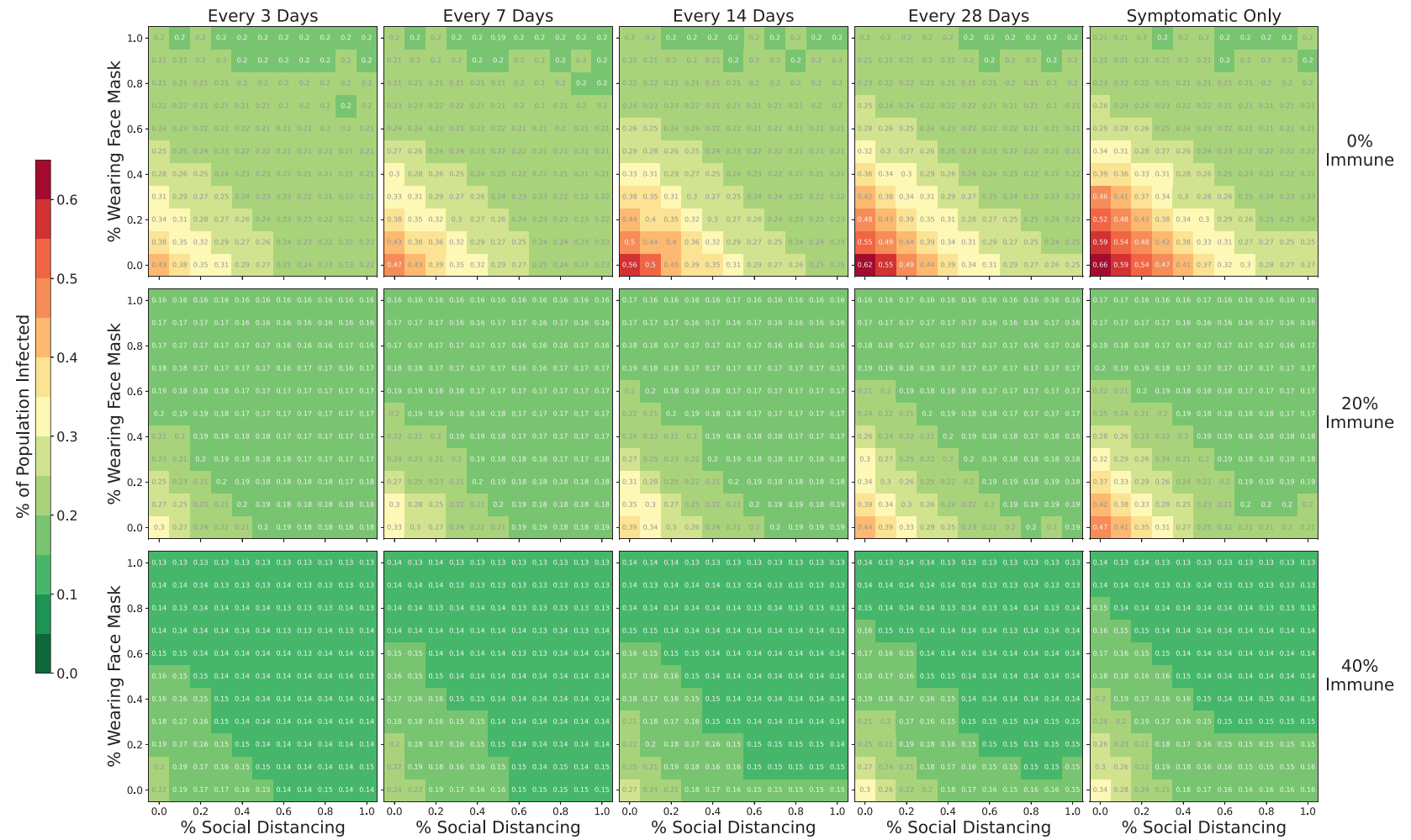

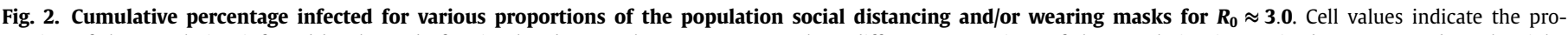

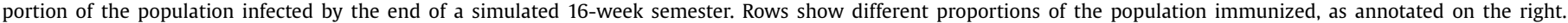
Columns show scenarios where scheduled testing was conducted every three, seven, 14 , and 28 days, respectively, and no scheduled testing.

are still facing an uncertain future. With bumpy vaccine roll-outs in some areas of the world and financial strains due to decreased enrollment, many colleges wonder how they will be able to return to some sort of normalcy and what steps they can take to accelerate the process (St. Amour, 2020). The 2015 United Nations Sustainable Development Goals aim to ensure both healthy lives and inclusive and equitable quality education (United Nations, 2015). In order to continue to advance toward these objectives, it is important to determine how schools can reopen safely, thereby allowing them to deliver quality education while promoting well-being.

In this study, we endeavored to help universities navigate the challenges of reopening by examining the efficacy of regularly scheduled (i.e., screening) testing in a residential college population in conjunction with various levels of immunization. We found that while testing should be an integral part of every university's mitigation strategy, moderate levels of immunity, mask wearing, and social distancing can allow universities the freedom to test less frequently. As such, if colleges can achieve high vaccination rates, they may be able to relax testing to just once or twice each month, saving valuable resources. While our focus is on a university setting, our methods and results could be applied to other residential environments.

In contrast to previous studies, we used a real-world contact network as the basis for our simulations, allowing us to capture the underlying heterogeneous social behavior of college students, which undoubtedly alters how the virus spreads. In addition, many previous studies in this area accounted for only a small number of implementation obstacles and compliance issues. We allowed for external infections, a background rate of influenza-like illness, time-dependent test sensitivity, and test result delays, as well as student non-compliance throughout the testing and isolation process.

The close proximity data we employed were collected long before the pandemic when students did not limit their interactions with others. Therefore, our data represent a worst case scenario where students do not reduce their contacts with others. However, when students reduce their contacts with others, they are effectively practicing a form of social distancing. Thus, these prepandemic contact patterns were necessary in order to study the effects of social distancing interventions, a goal of this study.

While vaccines do not provide perfect immunity, the mRNA vaccines currently being employed are highly effective at reducing infections and ostensibly effective at reducing transmission among those who become infected (Haas et al., 2021). Although we assumed perfect immunity in our simulations, our population immunity levels could be mapped to effective immunity levels in order to account for imperfect immunity and resultant breakthrough infections.

While our model incorporated many of the challenges we expect universities to face on campus, we did not explicitly model contact tracing. Although contact tracing can be an effective tool when implemented appropriately (Cencetti et al., 2021; Ferretti et al., 2020), it is often a resource-intensive undertaking, one that requires access to information on social contacts, access college students may be hesitant to grant. We also did not estimate the number of adverse outcomes expected to occur, although deaths and hospitalizations could be approximated from our results if additional assumptions are made. Finally, we did not incorporate any seasonal variation in the transmission of SARS-CoV-2, although new research suggests that in temperate climates transmission is significantly higher in the winter, when schools are in session, than in the summer (Gavenčiak et al., 2021). Thus, universities should consider their specific climate conditions when considering the disease burden they may face.

While this work provides novel insights into how immunization, repeat testing, isolation, and other strategies can reduce the spread of SARS-CoV-2 on college campuses, it is important to note that there is no one-size-fits-all approach (Bradley et al., 2020). 
The most successful schools will tailor their approach to their specific situation and adapt as circumstances change. Indeed, the most advantageous strategy may be an agile approach, one where testing frequency is adjusted based on the current transmission dynamics, an approach which has yet to be studied rigorously.

\section{Funding}

This study was supported by a Harvard University Department of Biostatistics scholarship and a U.S. Government scholarship (to $\mathrm{HH}$ ). Additional funding was provided by the Centers for Disease Control and Prevention (grant MInD: U01 CK000585 to RK) and the National Institutes of Health (NIAID R01 award AI138901 to JPO). The funding sources had no role in study design, data analysis, data interpretation, or the writing of the report.

\section{Ethical approval}

Separate medical ethical clearance not mandatory.

\section{Data sharing}

Proximity network data from the Copenhagen Network Study are in the public domain (https://doi.org/10.6084/m9.figshare. 7267433). All models and code for this project written in version 3.7 of the Python programming language are available through GitHub (https://github.com/onnela-lab/covid-campus).

\section{Contributors}

HH and JPO had full access to all data in the study. JPO conceptualized and supervised the study. HH, RK, and JPO contributed to the methodology, investigation, and visualization. HH drafted the manuscript with critical revision by RK and JPO. All authors have read and approved the final version.

\section{Declaration of Competing Interest}

The authors declare that they have no known competing financial interests or personal relationships that could have appeared to influence the work reported in this paper.

\section{Acknowlgedgments}

We thank Marc Lipsitch for his feedback on this project. We also thank Giang T. Nguyen and Max Wang for their critical reading of the manuscript.

\section{Supplementary material}

Supplementary material associated with this article can be found, in the online version, at doi:10.1016/j.ijid.2021.10.008

\section{References}

Alimohamadi Y, Taghdir M, Sepandi M. Estimate of the basic reproduction number for COVID-19: a systematic review and meta-analysis. Journal of Preventive Medicine and Public Health 2020:151.

Bradley EH, An MW, Fox E. Reopening colleges during the coronavirus disease 2019 (COVID-19) pandemic-one size does not fit all. JAMA Network Open 2020;3:e2017838.

Brook CE, Northrup GR, Ehrenberg AJ, Doudna JA, Boots M. Optimizing COVID-19 control with asymptomatic surveillance testing in a university environment. medRxiv; 2020.

Cencetti G, Santin G, Longa A, et al. Digital proximity tracing on empirical contact networks for pandemic control. Nature communications 2021;12:1-12.

Chang JT, Crawford FW, Kaplan EH. Repeat SARS-CoV-2 testing models for residential college populations. Health care management science 2020:1-14.

Christensen H, Turner K, Trickey A, et al. COVID-19 transmission in a university setting: a rapid review of modelling studies. medRxiv; 2020.

Davies NG, Abbott S, Barnard RC, et al. Estimated transmissibility and impact of SARS-CoV-2 lineage b.1.1. 7 in England. Science 2021;372.

Elbanna A, Wong GN, Weiner ZJ, et al. Entry screening and multi-layer mitigation of COVID-19 cases for asafe university reopening. medRxiv 2020.

Felter C. What to know about the global COVID-19 vaccine rollout so far. Council on Foreign Relations; 2021.

Ferretti L, Wymant C, Kendall M, et al. Quantifying SARS-CoV-2 transmission suggests epidemic control with digital contact tracing. Science 2020;368.

Gavenčiak T, Monrad JT, Leech G, et al. Seasonal variation in SARS-CoV-2 transmission in temperate climates. medRxiv; 2021.

Ghaffarzadegan N, Childs LM, TÄuber UC. Diverse computer simulation models provide unified lessons on university operation during a pandemic. BioScience; 2020.

Goyal R, Hotchkiss J, Schooley RT, De Gruttola V, Martin N. Evaluation of SARS$\mathrm{CoV}-2$ transmission mitigation strategies on a university campus using an agent-based network model. Clinical Infectious Diseases; 2021.

Gressman PT, Peck JR. Simulating COVID-19 in a university environment. Mathematical biosciences 2020;328:108436.

COVID-19 Scenario Modeling Hub. COVID-19 model projection. Round 5 2021;2021:05-28. Accessed https://covid19scenariomodelinghub.org/viz.html.

Haas EJ, Angulo FJ, McLaughlin JM, et al. Impact and effectiveness of mRNA BNT162b2 vaccine against SARS-CoV-2 infections and COVID-19 cases, hospitalisations, and deaths following a nationwide vaccination campaign in Israel: an observational study using national surveillance data. The Lancet; 2021.

Johansson MA, Quandelacy TM, Kada S, et al. SARS-CoV-2 transmission from people without COVID-19 symptoms. JAMA network open 2021;4:e2035057.

Lopman B, Liu CY, Le Guillou A, et al. A modeling study to inform screening and testing interventions for the control of SARS-CoV-2 on university campuses. Scientific reports 2021;11:1-11.

McPherson M, Smith-Lovin L, Cook JM. Birds of a feather: Homophily in social networks. Annual review of sociology 2001;27:415-44.

United Nations. General assembly resolutionn a/RES/70/1. Transforming Our World, the 2030 Agenda for Sustainable Development; 2015.

Paltiel AD, Zheng A, Walensky RP. Assessment of SARS-CoV-2 screening strategies to permit the safe reopening of college campuses in the united states. JAMA network open 2020;3:e2016818.

Sapiezynski P, Stopczynski A, Lassen DD, Lehmann S. Interaction data from the copenhagen networks study. Scientific Data 2019;6:1-10.

Shin M, Hickey K. Needs a little TLC: Examining college students emergency remote teaching and learning experiences during COVID-19. Journal of Further and Higher Education 2021;45:973-86.

St Amour M. Final fall enrollment numbers show pandemic's full impact. Inside Higher Ed; 2020.

Wikramaratna PS, Paton RS, Ghafari M, Lourenço J. Estimating the false-negative test probability of SARS-CoV-2 by RT-PCR. Eurosurveillance 2020;25:2000568. 